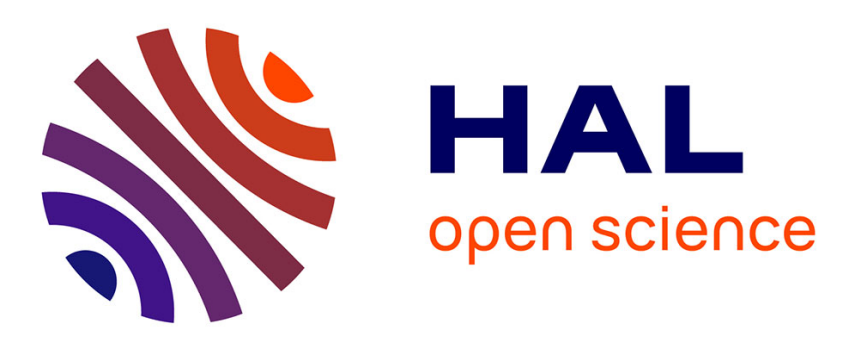

\title{
Improvement of Positioning Accuracy of PAMINSA (Parallel Manipulator of the I.N.S.A.)
}

\author{
Vigen Arakelian, Sébastien Briot
}

\section{To cite this version:}

Vigen Arakelian, Sébastien Briot. Improvement of Positioning Accuracy of PAMINSA (Parallel Manipulator of the I.N.S.A.). ROMANSY 2006, Jun 2006, Varsovie, Poland. hal-00451943

\section{HAL Id: hal-00451943 \\ https://hal.science/hal-00451943}

Submitted on 25 Jun 2019

HAL is a multi-disciplinary open access archive for the deposit and dissemination of scientific research documents, whether they are published or not. The documents may come from teaching and research institutions in France or abroad, or from public or private research centers.
L'archive ouverte pluridisciplinaire HAL, est destinée au dépôt et à la diffusion de documents scientifiques de niveau recherche, publiés ou non, émanant des établissements d'enseignement et de recherche français ou étrangers, des laboratoires publics ou privés. 


\title{
Improvement of Positioning Accuracy of PAMINSA (Parallel Manipulator of the I.N.S.A.)
}

\author{
Vigen Arakelian and Sebastien Briot \\ Département de Génie Mécanique et Automatique \\ Institut National des Sciences Appliquées (I.N.S.A.) de Rennes \\ 20 av. des Buttes de Coesmes, CS 14315 \\ F-35043 Rennes, France \\ e-mails : vigen.arakelyan@insa-rennes.fr \\ sebastien.briot@ens.insa-rennes.fr
}

\begin{abstract}
In this paper we present new design approaches for the positional accuracy improvement of the 4-DOF parallel manipulator PAMINSA. The particularity of this manipulator is the decoupling of displacements in the horizontal plane from its translation along the vertical axis. The positioning errors caused by the inclination of the platform relating to the base are cancelled by means of two different approaches. The first solution is realized by means of two correcting systems mounted on the drive system of the vertical displacements. The second solution is carried out by a correcting mass mounted on the platform. The suggested solutions are illustrated by the numerical examples and the performances of such a design are shown.
\end{abstract}

\section{Introduction}

It is well known that the parallel manipulators show a better stiffness than the serial manipulators. Their actuators and gearing are fixed, resulting in a low moving mass. Thus, the payloads capacity of parallel manipulators with lightweight links is more important than the serial manipulators. However they have nonlinear coupled kinematics and dynamics, which is a drawback for the industrial applications. It is obvious that the parallel structure with linear inputoutput equations is more appealing than the nonlinear one. In recent years, the decoupling of motions of parallel manipulators has attracted researchers' attention and different structures have been proposed. Previous works on this problem may be arranged into two principal groups: (i) fully decoupled parallel structures in which the architecture of the manipulator is such that its input/output equations are linear and (ii) position/orientation decoupled manipulators in which the end-effector's position is independent of the orientation.

One of the first studies devoted to the design of fully decoupled parallel structures was developed by Kong and Gosselin (2002). In this work, a class of 3-legged 3-DOF translational parallel manipulators with linear input-output equations was proposed. Another simple fully decoupled 3-DOF translational parallel mechanism named Tripteron was discussed in the paper Gosselin et al. (2004). Carricato and Parenti-Castelli (2004) proposed a novel parallel mechanism with fully decoupled degrees of freedom. This mechanism consists of two interconnected slider crank linkages, each of which independently actuates one of the Euler angles of the input link. 
Several types of translational parallel mechanisms with decoupled motions were discussed in the next work of the same authors (2004). The parallel robot, called T3R1, with four degrees of freedom and decoupled motions was developed at the French Institute of Advanced Mechanics (Bouzgarrou et al., 2004).

In spite of rather encouraging results, it should be noted that the fully decoupling of the motions also brings some disadvantages, such as for example, the increase of the number of joints and a loss of rigidity. It is obvious that it is difficult to solve the problem of the fully decoupling of the motions by keeping the many advantages of the parallel mechanisms. This is why we tried to find a compromise between the decoupling of the motions and the architectural characteristics of the parallel structures.

Another trend of the kinematic decoupling is proposed in the design of the PAMINSA, a new 4-DOF parallel manipulator that has been designed and developed at the I.N.S.A. ${ }^{1}$ in Rennes. It consists of decoupling the motions in the horizontal plane and the translation along the vertical axis.

\subsection{Description of the PAMINSA}

Fig. 1 shows a 3D model of the PAMINSA with three legs. Each leg of the manipulator is realized by a pantograph mechanism (Fig. 2) with two input points $A_{i}$ and $B_{i}$, and an output point $C_{i}(i=1,2,3)$. Each input point $A_{i}$ is connected with the rotating drive $M_{i}$ by means of the prismatic guide mounted on the rotating link.

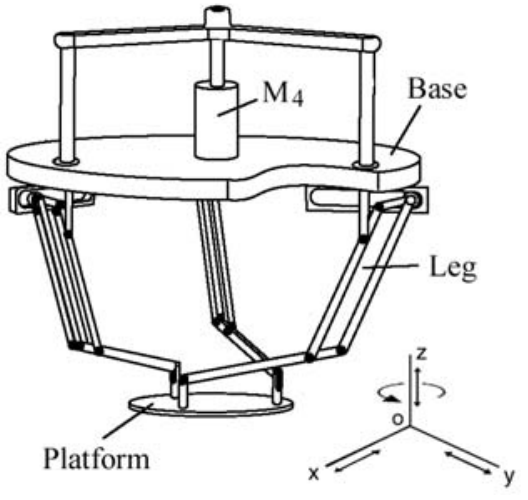

Figure 1. 3D view of the PAMINSA.

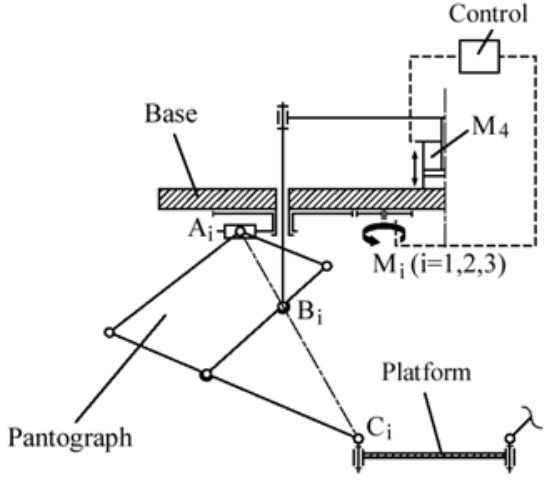

Figure 2. Schematics of each leg.

This type of manipulator architecture allows the generation of motion in the horizontal plane by the rotating actuators $\mathrm{M}_{1}, \mathrm{M}_{2}, \mathrm{M}_{3}$ and the vertical displacements by the linear actuator $\mathrm{M}_{4}$. Thus the displacements $(x, y, \theta)$ of the platform in the horizontal plane (xOy) are independent of the vertical displacements $z$.

In the concept of the PAMINSA, the following properties of the hand operated manipulators (see Arakelian, 1998) are used: the work of the gravitational forces of the manipulated object displaced in the horizontal plane is zero because the gravitational forces are always perpendicular

1 Institut National des Sciences Appliquées (I.N.S.A.) - National Institute of Applied Sciences. 
to the displacements. However, the work of the same forces in the case of the vertical displacements is different from zero (the gravitational forces are parallel to the displacements).

Thus, the rotating actuators move the platform in the horizontal plane and the work due to the gravity of the manipulated object is equal to zero. Static properties of PAMINSA were discussed in the study Arakelian et al. (2005), in which was shown that by using an optimal redistribution of masses of the pantograph linkages, the input torques of the rotating actuators of the PAMINSA can be cancelled. In the same study the analytical dynamic modeling of the suggested manipulator by means of Lagrange equations was achieved.

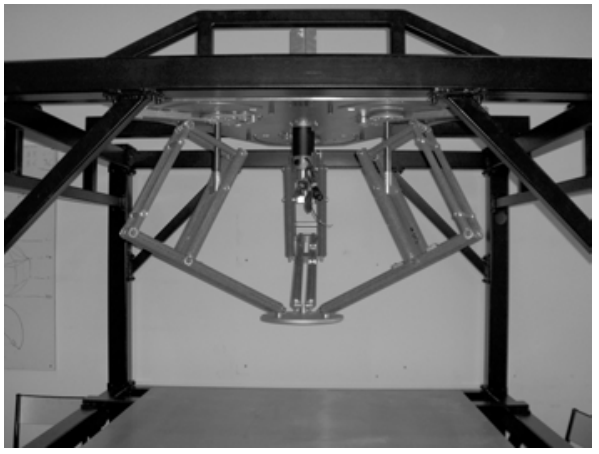

Figure 3a. The prototype of PAMINSA developed at the I.N.S.A. of Rennes.

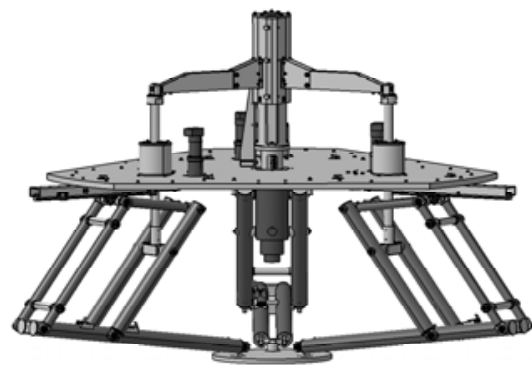

Figure 3b. The CAD model of the prototype.

Fig. 3 shows the prototype of the PAMINSA, which has been developed at the I.N.S.A. of Rennes. It should be noted that the proposed parallel structure has been patented (Arakelian et al., 2005).

\subsection{The obvious advantages of PAMINSA}

This manipulator has many advantages and it appears to us that the developed manipulator could be used in industrial applications for the manipulation of heavy equipments with great positioning accuracy. We would like to note briefly these advantages: a) a simplification of the vertical control based on the linear input/output equation; b) the improvement of the control in the horizontal plane because the loads of the gravitational forces of the platform on the rotating actuators have been cancelled; c) the improvement of positioning accuracy along the vertical axis because the mechanical locking of the structure does not allow the altitude variations during the displacements in the horizontal plane.

The letter property was discussed in the recent study Arakelian et al. (2005), in which the positioning errors along vertical axis and errors caused by the inclination of the moving platform taking into account the elasticity of links was analyzed. It was shown that the suggested manipulator allows the moving of the platform on the horizontal plane with great accuracy (about $20 \mu \mathrm{m}$ without charge and $140 \mu \mathrm{m}$ with a payload of $20 \mathrm{~kg}$ applied on the platform). However, PAMINSA has a very good load-carrying capacity and it can support much more. 
This contribution deals with the methods and new approaches for the improvement of positioning accuracy of PAMINSA.

\section{Improvement of positioning accuracy taking into account the elasticity of links}

Many industrial applications of parallel manipulators, such as the assembly of electronic, optical units, or several medical applications require high accuracy. It should be noted that most of parallel manipulators used today are much better at repeatability than at accuracy. For improvement of position accuracy of parallel manipulators, it is possible to use the calibration methods, to increase the rigidity of links or lack of backlashes in drive systems. A new approach called Geometric and Elastic Error Compensation (GEC) was proposed in the study of Meggiolaro and Dubowsky (2001). It was shown that the two techniques can be effectively combined to achieve high absolute positioning accuracy.

In this paper, for improvement of positioning accuracy of PAMINSA, we propose new compensation schemes, which consist of the introduction of complementary units into the initial system making it possible to cancel the positioning errors. Two different approaches are proposed and the performances of such a design are shown.

\subsection{Improvement of positioning accuracy of PAMINSA by means of correcting systems mounted on the drive system}

Most research papers devoted to the study of parallel manipulators deal with the mechanical structures with rigid links and without clearances in the joints. So in this case the position of the platform is considered perfectly parallel to the base. But in reality, the errors due to the elastic deformations of the mechanical structure of the manipulator change the position of the platform (attitude and inclination).

Static rigidity is defined as the $6 \times 6$ symmetrical matrix $\mathbf{K}$ that maps generalized infinitesimal displacements $\boldsymbol{\Delta} \boldsymbol{X}=\left[\begin{array}{llllll}\delta x & \delta y & \delta z & \delta \phi_{x} & \delta \phi_{y} & \delta \phi_{z}\end{array}\right]^{T}$ of the platform to generalized external loads $\boldsymbol{F}=\left[\begin{array}{llllll}F_{x} & F_{y} & F_{z} & M_{x} & M_{y} & M_{z}\end{array}\right]^{T}$.

Thus, we have

$$
\boldsymbol{F}=\boldsymbol{K} \cdot \Delta \boldsymbol{X}
$$

In order to characterize PAMINSA static rigidity, we map the infinitesimal displacements for the whole workspace. The obtained results show that the errors of the elastic deformations of the structure are relatively small. With a payload of $20 \mathrm{~kg}$ applied on the platform, the errors, caused by the rotation of the platform about the $x$ and $y$ axes are shown in Fig. 4. The analysis of the obtained results shows that the position in which the structure is less deformed is the central position. When the platform moves away from this position, the manipulator becomes less rigid and loses its accuracy.

These errors are less important if the output point $P$ (Fig.5), i.e. the end of a surgical device or a sensor, is located on the horizontal plane of the platform. But the error becomes more important if this output point is moved from the horizontal plan of the platform. For example, if the output point is located on the plane $x O z$ and it is moved away $200 \mathrm{~mm}$ from the horizontal 
plane of the platform (with $100 \mathrm{~mm}$ radius), the error $0.14 \mathrm{~mm}$ of the platform along the z-axis increases at the end of the output point until $0.57 \mathrm{~mm}$ (see Fig.5).

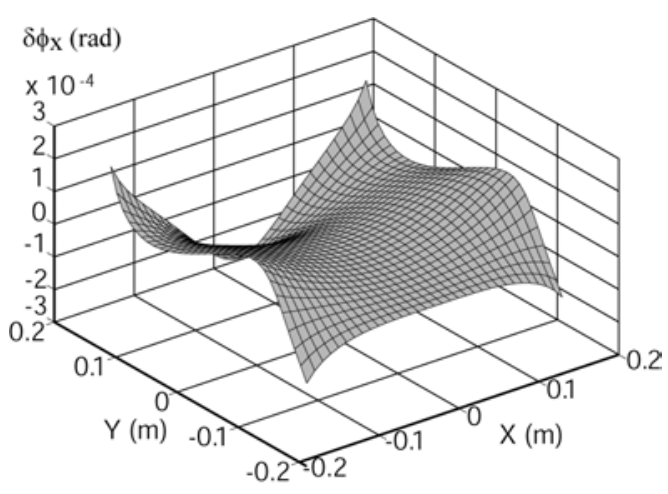

Figure 4a. Errors caused by the rotation of the platform about the $\boldsymbol{x}$ axis.

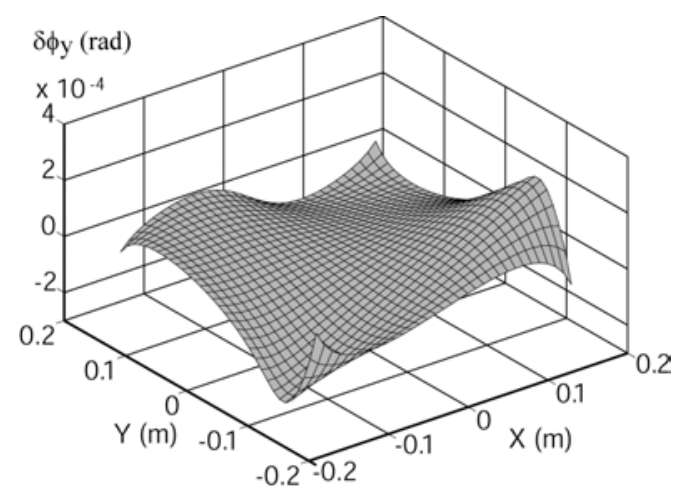

Figure 4b. Errors caused by the rotation of the platform about the $\boldsymbol{y}$ axis.

It is obvious that the improvement of positioning accuracy can be achieved by the increase of the rigidity of links. However it is also promising to develop the design methods for improvement of positioning accuracy by additional correcting systems.

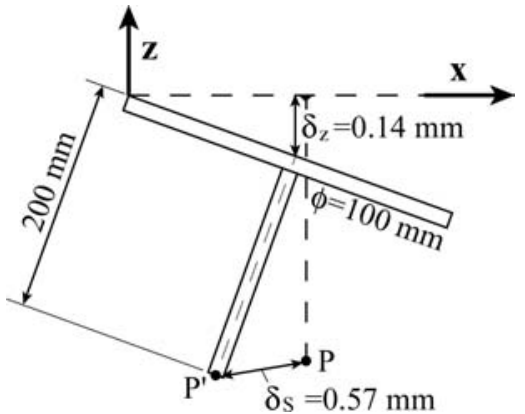

Figure 5. Absolute positioning errors of the output point, which is moved away $200 \mathrm{~mm}$ from the horizontal plane of the platform.
Fig. 6 shows PAMINSA with two compensation systems, which are presented in Fig.7. It should be noted that in the modified design of the manipulator the joints on the platform are also changed: the universal joints used in the initial version are replaced by the spherical pairs. The compensation systems, which cancel the errors due to the elasticity of links are provided with two complementary actuators $\mathrm{M}_{\mathrm{ci}}$. These actuators allow the displacements of the pantograph's points $B_{i}$ it making possible to eliminate the inclination error of the platform. These modifications allow the correction of the vertical positions of two spherical pairs of the platform, which is sufficient for the cancellation of the positioning error of the inclination of the platform.

The vertical positions of such a spherical pair located on the platform can be determined analytically for the whole workspace (or given altitude) of the manipulator on the base of equation (1) or by using three sensors mounted on the platform. The measuring of the spherical joint motion errors for the manipulators with three and six prismatic joints (for Tripod and Hexapod types), as well as several installation examples of the sensors were discussed in the study of Oiwa (2002). 


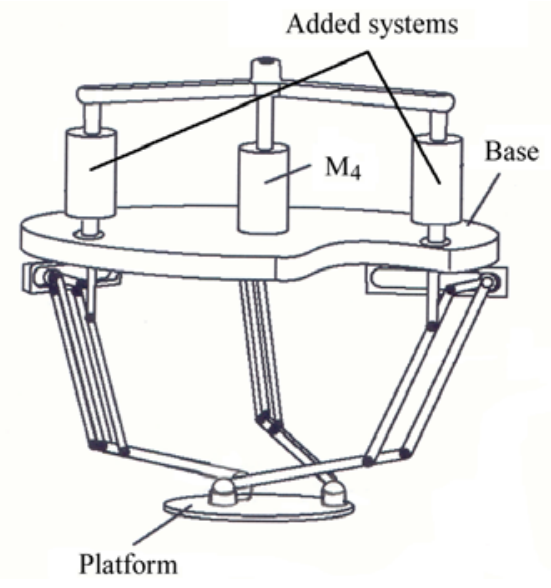

Figure 6. PAMINSA with added compensation systems.

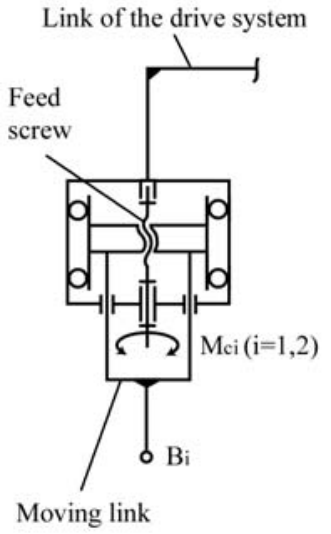

Figure 7. Kinematic schema of the added compensation systems for the correction of the positioning errors of the platform along the vertical axis.
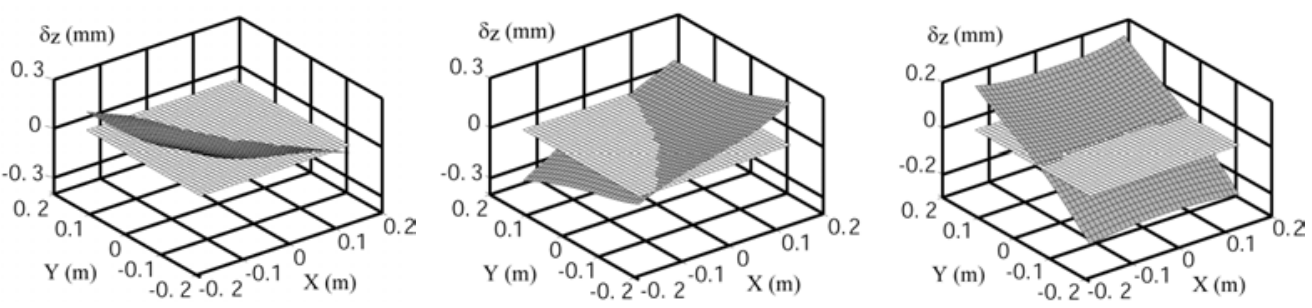

Figure 8. The vertical positioning errors of the platform's joints $C_{1}, C_{2}$ and $C_{3}$ of the initial and modified manipulators (the examined case correspond to the platform with orientation $\phi=0^{\circ}$ at the altitude $\mathrm{z}=-0.6 \mathrm{~m}$ ).

Note that, compensation scheme developed for PAMINSA is constructively more efficient because it is mounted on the drive system of the vertical displacements. As a result, the variable length of the compensation device is always vertical. It should be also noted that it is more simple for computation because the translational displacement in the added system can be found directly from positioning errors of the platform's joint, taking into account the magnification factor of the pantograph linkage. In this manner the significant reduction of errors can be achieved and the obtained results are shown in Fig.8. It is seen that after compensation of the elasticity errors of links the vertical positions of the platform's joints have constant values and the inclination error is eliminated. 


\subsection{Improvement of positioning accuracy of PAMINSA by means of correcting systems mounted on the platform}

Let us consider another correcting system mounted on the platform of PAMINSA. Such a system can be added on any parallel structure for the correction of positioning error of the inclination of the platform. The suggested system consists of a correcting mass, which has the possibility to turn about the vertical axis of the platform and to carry out the translational displacements on the horizontal plane (Fig.9).

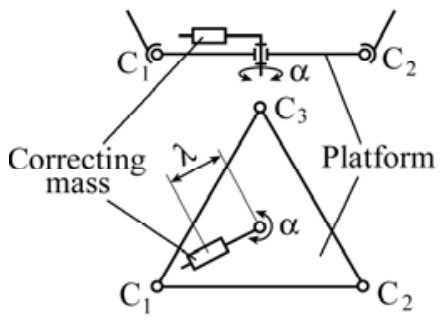

Figure 9. Kinematic schema of the correcting system mounted on the platform.
Introducing the correcting conditions into equation (1), we obtain

$$
\boldsymbol{\Delta} \boldsymbol{X}=\boldsymbol{K}_{s t}^{-1} \cdot \boldsymbol{F}+\boldsymbol{K}_{s t}^{-1} \cdot\left[\begin{array}{llllll}
0 & 0 & -m g & M_{s t(x)} & M_{s t(y)} & 0
\end{array}\right]^{T}
$$

From which taking into account that after correction

$$
\Delta \boldsymbol{X}=\left[\begin{array}{llllll}
\delta x & \delta y & \delta z & 0 & 0 & \delta \phi_{z}
\end{array}\right]^{T}
$$

we determine the static moments $M_{s t(x)}, M_{s t(y)}$ and then the position $\lambda$ and orientation $\alpha$ of the correcting mass $m$.

In other words the correcting mass $m$ should be located on the platform in such a manner that its gravity eliminated the inclination error of the platform.

For PAMINSA with parameters of the prototype (see Arakelian et al., 2005) the values of the position $\lambda$ and orientation $\alpha$ for the correcting mass $m=3 \mathrm{~kg}$ are shown in Fig. 10.
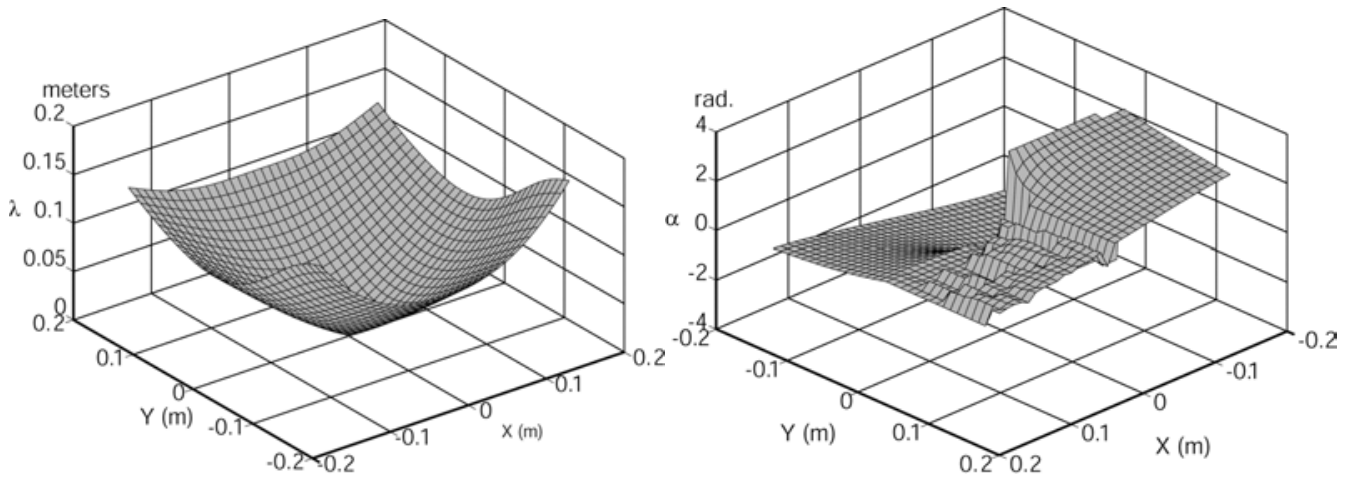

Figure 10. Position $\lambda$ and orientation $\alpha$ of the correcting mass $m$.

These values are obtained for the platform having the constant orientation $\phi=0^{\circ}$ at the altitude $z=-0.6 \mathrm{~m}$. The obtained results are the same as the previous case (Fig.8), i.e. after compensation of the elasticity errors of links, the vertical positions of the platform's joints have constant values and the platform becomes perfectly parallel to the base. 


\section{Conclusions}

In this paper, new design approaches for the improvement of positioning accuracy of 4-DOF parallel manipulator PAMINSA have been discussed. In general, the studies devoted to the parallel manipulators deal with the mechanical structures on the base of rigid body mechanics and consider that the platform is perfectly parallel to the base. Our previous works have shown that the elasticity of links has an influence on the positioning accuracy of the developed parallel manipulator. For cancellation of these positioning errors caused by the elasticity of links, two approaches have been presented. The first solution is realized by means of two correcting systems mounted on the drive system of the vertical displacements. The second solution is carried out by a correcting mass mounted on the platform. The obtained results show that after compensation of the errors caused by the elasticity of links, the vertical positions of the platform's joints have constant values and the inclination of the platform, relating to the base, is cancelled.

\section{Bibliography}

Kong, X. and Gosselin, C. M. (2002). A class of 3-dof translational parallel manipulator with linear input/output equations. Proceesings of the Workshop on Fundamental Issues and Future Research for Parallel Mechanisms and Manipulators, Québec City, Québec, Canada, 25-32.

Gosselin, C. M., Kong, X., Foucault, S. and Bonev, I. A. (2004). A fully decoupled 3-dof translational parallel mechanism. Parallel Kinematic Machines International Conference, Chemnitz, Germany, 595-610.

Carricato, M. and Parenti-Castelli, V. (2004). A novel fully decoupled 2-dof parallel wrist. The International Journal of Robotics Research, 23(6), 661-667.

Carricato, M. and Parenti-Castelli, V. (2004). On the topological and geometrical synthesis and classification of translational parallel mechanisms", Proceedings of the 11th World Congress in Mechanism and Machine Science, Tianjin, China, 1624-1628.

Bouzgarrou, B. C., Fauroux, J. C., Gogu, G., and Heerah, Y. (2004). Rigidity analysis of T3R1 parallel robot with uncoupled kinematics. Proceedings of the $35^{\text {th }}$ International Symposium on Robotics, Paris, France.

Arakelian, V. (1998). Equilibrage des manipulateurs manuels, Mechanism and Machine Theory, 33(4), 437-442.

Arakelian, V., Guegan, S., Briot, S. (2005). Static and dynamic analysis of the PAMINSA, Proceedings of the ASME 2005 IDETC/CIE Conference, September 24-28, Long Beach, California USA.

Arakelian, V., Maurine, P., Briot, S., Pion, E. (2005) Parallel robot comprising means for setting in motion a mobile element split in two separate subassemblies, WO2006021629, 2006-03-02.

Arakelian, V., Guegan, S., Briot, S., J. Le Flecher (2005). Design and prototyping of new 4, 5 and 6 degrees of freedom parallel manipulators based on the copying properties of the pantograph linkage. Proceedings of the $36^{\text {th }}$ International Symposium on Robotics, November 29 - December 1, Tokyo, Japan.

Meggiolaro M. and Dubowsky S. Improving the positioning accuracy of powerful manipulators with application in nuclear maintenance (2001). Anais do 16 Congresso Brasileiro de Engenharia Mecânica (COBEM), Robotics and Control, Vol. 15, pp. 210-219, ABCM, Uberlândia.

Oiwa T. Accuracy improvement of parallel kinematic machine (2002). Proceedings of the 6th International Conference on Mechatronics Technology, September 29 - October 3, Kitakyushu, JAPAN. 